\title{
Depth separation and lateral interference
}

\author{
ROBERT FOX and ROBERT PATTERSON \\ Vanderbilt University, Nashville, Tennessee 37240
}

\begin{abstract}
In four experiments, the perceptual interaction between an annulus and a Landolt $C$ enclosed within it was investigated as a function of their perceived relative depth positions and of the perceived lateral distance between the inner edge of the annulus and the outer edge of the C. To permit facile and unconfounded manipulation of perceived depth, the stimuli were stereoscopic contours formed from dynamic random-element stereograms. Either one or both stimuli were visible continuously. The effect of the annulus on the Landolt $C$ was assessed by forced-choice recognition thresholds of the $\mathrm{C}$ and by judgments of its apparent clarity. The main results were: (1) For both threshold recognition and apparent clarity, perceived depth separation has a strong effect on the strength of perceptual interaction; (2) the effect is asymmetrical in that the stimulus perceived as in front of its partner and closer to the observer has greater perceptual potency; and (3) as spacing between the elements increases, perceptual interaction declines independently of depth position. The implications of these data for general theories of stimulus interaction in three-dimensional space are discussed.
\end{abstract}

Lateral interference is a general term intended to encompass many different kinds of perceptual interactions between continuously visible, suprathreshold stimuli that lie in the same depth plane (z-axis) and are in close spatial proximity ( $\mathrm{x}$ - and $\mathrm{y}$-axes). The key observation from which the notion of interference derives is that a stimulus surrounded by others is less perceptible than when it is seen in isolation. A ubiqquitous example of such interference, described by Woodworth (1938), is provided by horizontal arrays of alphabet letters-letters at the end of the string are more perceptible than embedded ones flanked by partner letters. A second example, from the ophthalmic and optometric literature, is the phenomenon known as crowding. In the well-known clinical test of visual acuity known as the Snellen Chart, randomly ordered rows of letters are reduced in size in discrete steps; at a size at which letters within the row are not resolvable, each letter can be seen if presented alone.

Two well-known phenomena closely related to lateral interference are simultaneous contrast and visual masking. In simultaneous contrast, the brightness of a surface can be markedly reduced if it is adjacent to or surrounded by a surface of a higher intrinsic brightness. In visual masking, the perceptibility of transient stimuli is reduced if they occur closely coupled in space and in time.

Theoretical treatments of all these phenomena have regarded them as manifestations of a common

Support for this investigation was provided by Grants EY 00590-17 from the National Institutes of Health and by N0001476-C-1101 from the Office of Naval Research. Requests for reprints should be sent to Robert Fox, Department of Psychology, Vanderbilt University, Nashville, Tennessee 37240. underlying lateral inhibitory process, the notion of which is derived from the physiological concept of lateral inhibition (e.g., Cornsweet, 1970). This approach considers interaction only in $x$ - and $y$-axes and fails to consider the depth position of the stimuli. This failure reflects the widespread assumption that information about the characteristics of stimuli are analyzed first by the visual system. This information is then used (i.e., it provides cues) to determine the depth location of stimuli.

But an alternative position, explicit within the Gestalt tradition (e.g., Koffka, 1935), posits an analog representation of visual space within the visual system. This theoretical position assumes that depth information is processed either simultaneously or prior to the processing of stimulus information. According to this view, interaction and interference would not occur between stimuli sufficiently separated in depth. Some tests of that hypothesis have been made by manipulating cues so that physical stimuli appear to lie at different perceived distances. In the main, the results support this hypothesis (much of the work is reviewed by Gilchrist, 1980; Gogel, 1978; Lehmkuhle \& Fox, 1980). But a full test of the hypothesis that stimulus interaction and interference depend upon depth position is impeded by the intrinsic difficulty in manipulating the apparent depth of physical stimuli without introducing potentially confounding differences in retinal stimulation.

An approach that avoids the problem of confounding stimulation while at the same time permitting facile manipulation of large changes in apparent depth is the use of stereoscopic contours generated from random-element stereograms (e.g., Julesz, $1971,1978)$. In a functional sense, these contours bypass the more peripheral stages of the visual sys- 
tem and arise at the central stages devoted to stereopsis. Even though they do not exist as physical luminance gradients impinging on the retina, they can induce illusions, aftereffects, and other perceptual phenomena similar to those produced by physical contours.

In most of the prior research using random-element stereograms, only static versions (e.g., photographs) were available; hence, parameters of the stereoscopic configuration were fixed. But recent technological developments have made it possible to generate dynamic random-element stereograms that permit the parameters of the stereoscopic display to be changed instantaneously without introducing monocular cues. The system for generating dynamic random-element stereograms employed in the present study has been developed and used in a variety of research applications (e.g., Fox, Lehmkuhle, \& Bush, 1977; Fox, Lehmkuhle, \& Leguire, 1978; Staller, Lappin, \& Fox, 1980). A description of the electronic portion of the generation system is given in Shetty, Brodersen, and Fox (1979).

This dynamic stereogram system was used by Lehmkuhle and Fox (1980) to investigate the effect of depth separation on visual metacontrast masking. The test stimulus was a stereoscopic contour, configured as a Landolt $C$, whose gap position could be varied to obtain forced-choice recognition thresholds. The masking stimulus was a briefly presented annulus that surrounded the Landolt $C$ and varied in depth position and onset time relative to it. The main results were as follows: When test and mask occupied the same perceived depth position, considerable masking was obtained. When the depth position of the test was perceived as in front of the mask and closer to the observer, masking declined as a function of increases in depth position between test and mask. When the perceived depth positions were reversed and the mask appeared in a depth plane in front of the test and closer to the observer, masking was enhanced relative to the case in which both occupied the same perceived depth plane. Masking was clearly influenced by perceived depth between test and mask, but the asymmetrical nature of the depth-dependent relationship, which was termed the front effect, was unexpected. A tentative hypothesis advanced to account for it is based on the notion that whenever objects occupy similar and, possibly, competing visual directions (similar $x / y$ positions), the stimulus closer to the observer is processed with greater facility or given greater weight by the visual system.

The present experiments were inspired directly by the Lehmkuhle and Fox investigation and were designed to determine: (1) whether the pattern of results obtained with visual masking was restricted to the perceptual interaction of transient stimuli or whether it also would be obtained when one or both of the interacting elements were not transient (i.e., under conditions in which lateral interference might occur); and (2) whether the interaction would be influenced by perceived lateral distance between stimuli. On the hypothesis developed to account for the front effect, interaction should decline as perceived lateral distance-or, alternatively, the difference in visual direction between stimuli-increases.

\section{GENERAL METHOD}

\section{Apparatus}

Only a brief overview of the system developed for the dynamic generation of random-element stereograms will be given here, since this system has been discussed in some detail elsewhere (e.g., Lehmkuhle \& Fox, 1980; Shetty et al., 1979). The system consists of two functional units, the display device and the electronic generation unit. For this application, however, an additional compo-, nent, the optical programming device, has been added.

The display device is a color television modified so that the red and green guns can be electronically controlled at the level of the video amplifiers, with the blue gun being turned off. Modulation of the red and green guns produces random-dot matrices composed of red and green dots. When appropriately matched red and green filters are placed before the eyes of an observer, the matrices stimulate separate eyes and thereby fulfill the requirements of stereoscopic viewing. This is the well-known anaglyph method of stereoscopic presentation.

The electronic unit that modulates the red and green guns is a hardwired device made from high-speed integrated circuits. It drives the guns in the raster-scan mode at standard video frequencies. There are four subsystems that are assigned particular functions: (1) The undelayed-dot generation system generates random matrices of red and green dots without disparity that would, if displayed, completely fill the screen. (2) The size-shape system specifies the $x / y$ coordinates of the stereoscopic form to be displayed. This is done by blanking the red and green dots produced by the undelayed-dot generation system. If the output of the sizeshape system were to be displayed, one would see a black shape on the screen corresponding to the size and shape of the to-bedisplayed stereoscopic form surrounded by red and green dots from the undelayed-dot generation system. (3) The dot-delay system produces a slight delay in the output of one or the other of the electron guns, resulting in a difference in spatial position between red and green dots. It is this spatial displacement that provides the retinal disparity essential for stereopsis. The only dots that are delayed are those that fill in the area specified by the size-shape system. When undelayed dots from the other gun also fill in the area, a disparity exists between the delayed dots and the undelayed dots. If the output of all previous stages were to be displayed at this point, one would perceive the stereoscopic form but a vertical gap at one end of the form would also be visible. (4)The gap-filling system provides dots without disparity, which precisely fill in the gap produced by introducing the delay. When all outputs of the systems are combined, by ANDing logic operations, and simultaneously displayed on the television screen, the stereoscopic form can be seen without the presence of monocular cues. It should be noted that in this method of stereogram generation the size of the stereoscopic form remains independent of disparity, thereby avoiding the confounding of size and disparity inherent in some methods of stereogram construction. As Bridgman (1964) and Gulick and Lawson (1976) have pointed out, the practice of filling the gap at one side of the shifted matrix with elements from the opposite side produces columns of unpaired elements whose numbers increase with disparity. This results in a reduction in the width of the stereoscopic form that covaries with disparity, a reduction that is due to the physical characteristics of the stereo- 
gram and not related to an apparent reduction induced by size constancy. In this dynamic mode of operation, all dots in the stereogram are replaced, with their positions assigned randomly, by a random generator at either the field rate $(60 \mathrm{~Hz})$ or the frame rate $(30 \mathrm{~Hz})$ of the video receiver. Dot replacement produces a continual apparent motion of the dots; this motion, however, does not impair the visibility of the stereoscopic form and permits the stereoscopic form to be continuously changed in $\mathrm{x}, \mathrm{y}$, and $\mathrm{z}$ positions without introducing monocular cues.

The electronic generation unit, by itself, allows only for the generation of rectilinear stereoscopic forms. To overcome this restriction, the optical programming device makes it possible to present, as a stereoscopic form, virtually any black-and-white twodimensional stimulus configuration. The scan of a modified blackand-white video camera is synchronized with the sweep of the video receiver. The analog voltage emitted by the camera, which varies as it sweeps over contours varying in luminance, is digitized and used to specify the area that is to receive the delayed dotsin effect, it controls the size-shape system. The number of configurations that could be presented simultaneously is governed by the number of cameras used. The parameters of the stereoscopic configuration encoded by one camera, such as depth position and exposure duration, can be manipulated independently of the parameters of the stereoscopic configuration encoded by another camera. A timing device provides for precise control of the duration of exposure of each stereoscopic configuration. Durations of exposure are always multiples of either the field or frame rate of the receiver.

In the present experiments, two kinds of stimuli were used-a Landolt $C$ (test figure) and an annulus that surrounded the $C$. Of the two video cameras employed in these experiments, one scanned achromatic, two-dimensional images of the Landolt $\mathrm{C}$, and the other scanned similar images of the annulus. In all experiments, the observer viewed the display, which was a table-model color television receiver, under constant conditions-fixed viewing distance and stable head position.

\section{THRESHOLD RECOGNITION EXPERIMENTS}

The purpose of Experiments 1 and 2 was to investigate the influence of perceived depth separation (Experiment 1) and perceived lateral separation (Experiment 2) on the lateral interference between a briefly presented test stimulus, the Landolt $C$, and a continuously visible annulus. In these experiments, perceptual interference was measured by obtaining two-alternative forced-choice recognition thresholds for the gap position of the Landolt C.

\section{Method}

\section{Stimuli}

The Landolt $C$ subtended an angle of $1.53 \mathrm{deg}$ at the eye, with the gap subtending an angle of $.64 \mathrm{deg}$. The width of the annulus was always $1.83 \mathrm{deg}$. The lateral separation between the inner edge of the annulus and the Landolt $C$ was .36 deg in Experiment 1 and either .36 or $1.27 \mathrm{deg}$ in Experiment 2. Thus, in all cases the annulus surrounded, but did not overlap, the $C$.

\footnotetext{
Observers

Five persons, all graduate students at Vanderbilt University, were paid to serve in Experiment 1. Of those five, three observers subsequently served in Experiment 2. All had normal or correctedto-normal acuity, good stereoacuity, and considerable experience in detecting stereoscopic stimuli constructed from dynamic randomelement stereograms. All observers were naive with regard to the hypotheses under consideration.
}

\section{Experiment 1}

To examine the influence of perceived depth separation on lateral interference, Experiment 1 employed one value of lateral separation between the Landolt $\mathrm{C}$ and annulus-namely, $.36 \mathrm{deg}$ - and seven viewing conditions. Two of these were control conditions in which the Landolt $\mathrm{C}$ was presented without the annulus. One control condition was presented at the beginning (presession control) and the other at the end (postsession control) of each experimental session. These conditions provided a check on the stability of baseline thresholds. The other five conditions were the disparity conditions, in which the apparent depth position of the annulus relative to that of the Landolt $C$ was varied, with the annulus appearing in front of or in back of the $\mathrm{C}$ or occupying the same depth plane. Under all conditions, all disparities were crossed and the stereoscopic stimuli appeared in depth in front of the background elements. In all conditions, the Landolt $C$ was located at a disparity of $21 \mathrm{~min} 24 \mathrm{sec}$ from background. In the disparity conditions, the annulus was placed at one of five depth positions relative to the $\mathrm{C}$. In two conditions, the annulus appeared behind the Landolt $\mathrm{C}$, at disparities of $9 \mathrm{~min} 10 \mathrm{sec}$ and $15 \mathrm{~min} 17 \mathrm{sec}$ from background. In another condition, the annulus and $C$ appeared in the same depth plane, at a disparity of $21 \mathrm{~min} 24 \mathrm{sec}$ from background. In two other conditions, the annulus appeared in front of the Landolt $\mathrm{C}$, at disparities of $27 \mathrm{~min} 30 \mathrm{sec}$ and $33 \mathrm{~min} 37 \mathrm{sec}$ from background. It should be noted that simultaneous contrast between the annulus and $\mathrm{C}$ did not occur; this phenomenon does not seem to be a property of random-element stereograms. The display was situated $225 \mathrm{~cm}$ from the observers.

\section{Procedure}

This experiment used five trained observers, whose levels of performance reached asymptotic values prior to final data collection. The observers received considerable practice in forced-choice recognition of the gap position of the Landolt $C$, which varied randomly, with the restriction of equal occurrence between the 3:00 and 9:00 o'clock positions, across trials. Feedback was given after every trial. Exposure duration was varied as performance improved so as to maintain baseline performance within the range of $75 \%-90 \%$ correct responses; this level of performance was achieved with exposure durations ranging from 64 to $128 \mathrm{msec}$, with each duration adjusted for each observer.

Formal data collection involved five daily sessions in which the annulus was introduced and its depth position varied randomly for each observer over the five disparity conditions. For four of the five observers, 100 trials were presented under each of the seven viewing conditions; for the remaining observer, only 60 trials were presented under each condition due to time constraints. Changes in the apparent size of the annulus produced by size constancy were controlled by varying the size of the image scanned by the optical programming device, so that apparent size remained constant over all disparity condittons. This compensation did not alter the perceived lateral distance between the inner contour of the annulus and the outer contour of the Landolt $C$. 


\section{Results}

Figure 1 shows mean percent recognition for the control and disparity conditions. A one-way analysis of variance for repeated measures revealed a significant effect of depth position upon percent recognition $[F(6,24)=40.16, p<.0001]$. Duncan's multiple range test was employed in a post hoc analysis to compare recognition performance under the control and disparity conditions. The following differences were found to be significant: presession and postsession control conditions, and disparity conditions $9 \mathrm{~min}$ $10 \mathrm{sec}, 15 \mathrm{~min} 17 \mathrm{sec}$, and $21 \mathrm{~min} 24 \mathrm{sec}$ vs. disparity condition $33 \mathrm{~min} 37 \mathrm{sec}$; presession and postsession control conditions, and disparity conditions $9 \mathrm{~min}$ $10 \mathrm{sec}$ and $15 \mathrm{~min} 17 \mathrm{sec}$ vs. disparity conditions $21 \mathrm{~min} 24 \mathrm{sec}$ and $27 \mathrm{~min} 30 \mathrm{sec}$; and presession and postsession control conditions vs. disparity condition $15 \mathrm{~min} 17 \mathrm{sec}$ (for all comparisons, $\mathrm{p}<.01$ ). It can be seen from Figure 1 that, relative to the control conditions, the presence of the annulus in the equal depth condition did impair recognition performance for the Landolt C. Inspection of Figure 1 reveals an asymmetry of interference following depth manipulations of the annulus: Recognition performance improved when the annulus was moved from the plane of the Landolt $\mathrm{C}$ to positions behind the $\mathrm{C}$, but when it was

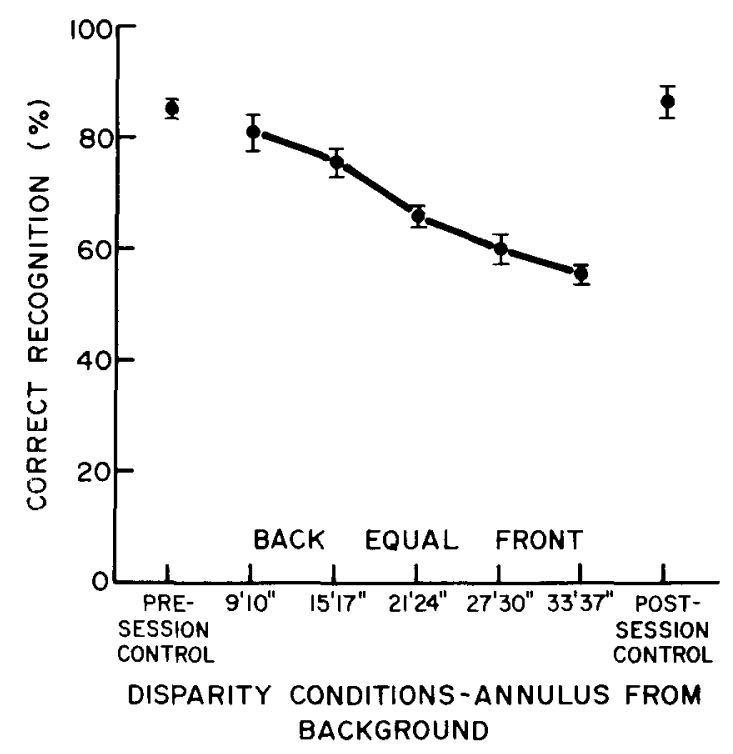

Figure 1. Forced-choice recognition thresholds for pre- and postsession control conditions, in which the Landolt $\mathbf{C}$ test figure was presented without the annulus, and for five disparity conditions in which the Landolt $C$ and annulus were presented together (the Landolt $C$ was near threshold, and the annulus was suprathreshold). "Back," "front,"' and "equal" indicate the following: For disparity conditions 9 min $10 \mathrm{sec}$ and $15 \mathrm{~min} 17 \mathrm{sec}$, the annulus was positioned behind the Landolt $C$, and for disparity conditions $27 \mathrm{~min} 30 \mathrm{sec}$ and $33 \mathrm{~min} 37 \mathrm{sec}$, the annulus was positioned in front of the Landolt C. For disparity condition 21 min $24 \mathrm{sec}$, both Landolt $C$ and annulus occupied the same depth plane. The value of lateral separation employed was $.36 \mathrm{deg}$. moved to positions in front of the $\mathrm{C}$, performance became worse.

\section{Experiment 2}

Experiment 1 demonstrated that lateral interference between the Landolt $C$ and annulus, as measured by threshold recognition, is significantly influenced by their relative depth positions. The lateral separation between the two stimuli also should affect interference, and Experiment 2 measured the effect of lateral separation on threshold recognition. Three viewing conditions were employed-one control condition and two lateral separation conditions in which the degree of lateral separation between the Landolt $C$ and annulus was varied. The same twoalternative forced-choice task used in Experiment 1 . was employed. In the two separation conditions, the relative depth positions of the Landolt $C$ and annulus remained equal at a disparity value of $21 \mathrm{~min} 24 \mathrm{sec}$ from background. The two values of lateral separation between the inner edge of the annulus and the $C$ were $.36 \mathrm{deg}$, hereafter referred to as the small separation condition, and $1.27 \mathrm{deg}$, hereafter referred to as the large separation condition.

\section{Procedure}

Three observers from Experiment 1 participated in the present experiment. Similar to Experiment 1, exposure durations of the $C$ were varied individually so as to maintain baseline threshold at a level of $75 \%-90 \%$ recognition for each observer (durations ranged from $48-128 \mathrm{msec}$ ). Three daily sessions were run, and a total of 150 trials was presented under each viewing condition; the order of the conditions was randomized for each observer every session.

\section{Results}

Figure 2 shows percent recognition for the control and two lateral separation conditions. A one-way analysis of variance for repeated measures found that the effect of lateral separation on percent recognition is reliable $[F(2,4)=67.75, p<.01]$. Duncan's multiple range test revealed the following differences to be significant: the control condition and the large separation condition vs. the small separation condition $(p<.01)$. As revealed in Figure 2, comparison of results from the control and small separation conditions shows that the presence of the annulus in a depth plane equal to that of the Landolt $\mathrm{C}$ did produce a decrement in recognition performance for the C. This decrement was specific to the small separation condition, however, as performance under the large separation condition was equivalent to that under the control condition.

\section{CLARITY RATINGS EXPERIMENTS}

The purpose of Experiments 3 and 4 was to examine the influence of both perceived depth separa- 


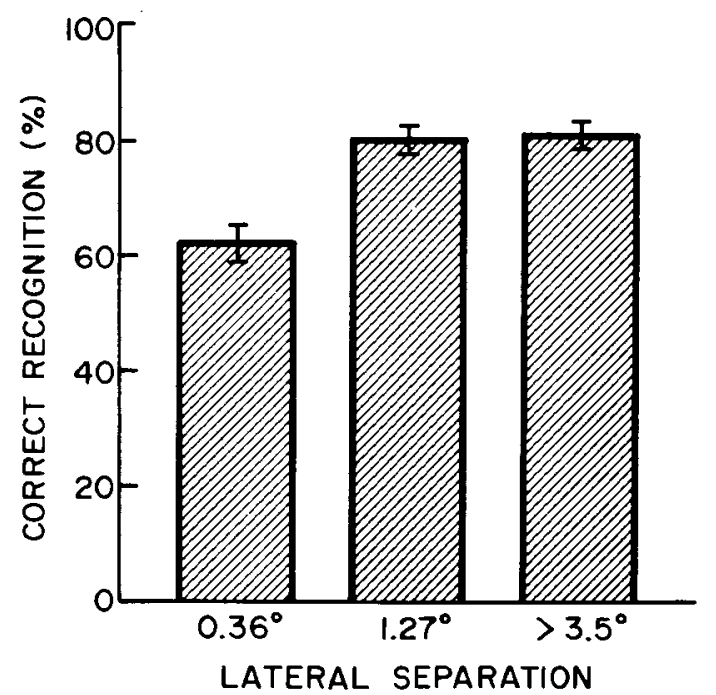

Figure 2. Forced-choice recognition thresholds for the 3.5-deg control condition, in which the Landolt $\mathbf{C}$ test figure was presented without the annulus, and for the .36- and 1.27-deg lateral separation conditions in which the Landolt $C$ and annulus were presented together in the same depth plane (the Landolt $C$ was near threshold, and the annulus was suprathreshold). Note that in the control condition, the lateral separation between the Landolt $\mathbf{C}$ and the nearest contour that may have interacted with the C-namely, the edge of the display - was greater than 3.5 deg.

tion (Experiment 3) and perceived lateral separation (Experiment 4) on the lateral interference between two continuously visible, suprathreshold stimuli-the Landolt $C$ and the annulus. In these experiments, perceptual interference was measured by obtaining ratings of apparent clarity of the Landolt $C$ from the observers. In this situation, changes in apparent clarity could not result from alterations in proximal stimulation or from motion parallax since stereoscopic percepts do not yield parallactic information. Lateral movements of the head cause the stereoscopic forms to appear to move with the observer in the same direction, so that, in the present experiments, the Landolt $C$ would still appear centered within the annulus.

\section{Method}

Stimuli

The Landolt C subtended an angle of $1.74 \mathrm{deg}$, and the gap subtended an angle of $.88 \mathrm{deg}$. The width of the annulus was always $3.52 \mathrm{deg}$. The lateral separation between the inner edge of the annulus and the $C$ was $.90 \mathrm{deg}$ in Experiment 3 and .90, 1.39, 1.91 , or $2.43 \mathrm{deg}$ in Experiment 4.

\section{Observers}

Four observers, three graduate students and one postdoctoral fellow at Vanderbilt University, were paid for their voluntary participation in Experiments 3 and 4. All four had normal or corrected-to-normal acuity, normal stereoacuity, and experience in perceiving stereoscopic stimuli constructed from dynamic random-element stereograms. All four observers were also naive with regard to the hypotheses under test.

\section{Experiment 3}

The lateral separation between the inner edge of annulus and $C$ employed in Experiment 3 was $.90 \mathrm{deg}$. Of the seven viewing conditions used in Experiment 1, only six conditions (one control and five disparity) were employed in Experiment 3. The precise values of disparity employed in the present experiment were the following: In all conditions, the Landolt $\mathrm{C}$ was located at a disparity of $20 \mathrm{~min} 0 \mathrm{sec}$ from background. For the two disparity conditions in which the annulus appeared behind the $C$, the annulus was positioned at disparities of $6 \mathrm{~min} 40 \mathrm{sec}$ and $13 \mathrm{~min}$ $20 \mathrm{sec}$ from background. For the condition in which the annulus and $C$ appeared in the same depth plane, they were both positioned at disparity $20 \mathrm{~min} 0 \mathrm{sec}$ from background. For the conditions in which the annulus appeared in front of the $C$, the annulus was positioned at disparities of $26 \mathrm{~min} \mathbf{4 0} \mathrm{sec}$ and $33 \mathrm{~min}$ $20 \mathrm{sec}$ from background.

\section{Procedure}

The clarity ratings were obtained in the following way: The observers were required to rate the perceived clarity of the Landolt C on a 7-point scale, with 1 indicating "not clear" and 7 indicating "very clear." The Landolt C was visible continuously, with the orientation fixed in the 9:00 o'clock position. The control and disparity conditions, three trials each, were presented randomly to the observers. As in Experiment 1, apparent size of the annulus remained constant throughout all depth manipulations.

\section{Results}

Figure 3 shows the observers' ratings of perceived clarity for the one control and five disparity conditions. A one-way analysis of variance for repeated measures revealed a significant effect of disparity conditions upon clarity ratings $[F(5,15)=7.4, p<.001]$. Duncan's multiple range test found the following differences to be reliable: the control condition vs. disparity conditions $13 \mathrm{~min} 20 \mathrm{sec}, 26 \mathrm{~min} 40 \mathrm{sec}$, and $33 \mathrm{~min} 20 \mathrm{sec}$, and disparity condition $6 \mathrm{~min} \mathbf{4 0 \mathrm { sec }} \mathrm{vs}$. disparity condition $33 \mathrm{~min} 20 \mathrm{sec}(p<.01)$; the control condition vs. disparity conditions $6 \mathrm{~min} 40 \mathrm{sec}$ and $20 \mathrm{~min} 0 \mathrm{sec}$, and disparity conditions $13 \mathrm{~min} 20 \mathrm{sec}$ and $20 \mathrm{~min} 0 \mathrm{sec}$ vs. disparity condition $33 \mathrm{~min} 20 \mathrm{sec}$ $(p<.05)$. It can be seen from Figure 3 that rated clarity was less when the annulus was presented with the Landolt $\mathrm{C}$ in the equal depth condition than when it was presented alone in the control condition. Moreover, an asymmetry of interference following depth manipulations of the annulus can be seen. Rated clarity was greater when the annulus was moved from the plane of the Landolt $\mathrm{C}$ to positions behind the $C$; yet, when moved to positions in front of the $C$, rated clarity was less. 


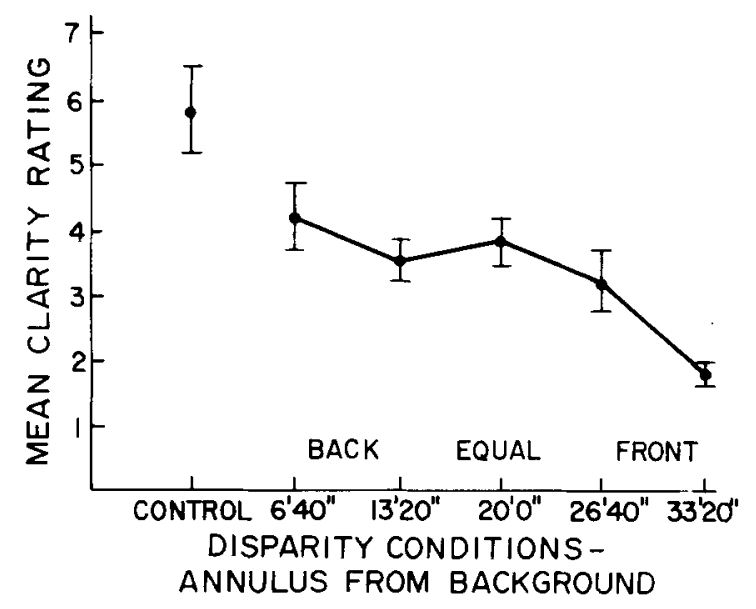

Figure 3. Clarity rating scores for the control condition, in which the Landolt $\mathbf{C}$ test figure was presented in isolation, and for five disparity conditions in which the Landolt $C$ and annulus were presented together (both Landolt $C$ and annulus were suprathreshold). "Back," "front," and "equal" indicate the following: For disparity conditions 6 min $40 \mathrm{sec}$ and $13 \mathrm{~min} 20 \mathrm{sec}$, the annulus was positioned behind the $L$ andolt $C$, and for disparity conditions $26 \mathrm{~min} 40 \mathrm{sec}$ and $33 \mathrm{~min} 20 \mathrm{sec}$, the annulus was positioned in front of the Landolt C. For disparity condition $20 \mathrm{~min} 0 \mathrm{sec}$, both Landolt $C$ and annulus occupied the same depth plane. The value of lateral separation employed was $.90 \mathrm{deg}$.

\section{Experiment 4}

To examine the influence of perceived lateral separation on lateral interference as measured by clarity ratings, Experiment 4 employed three values of lateral separation between the inner edge of the annulus and the Landolt C: $1.39,1.91$, and $2.43 \mathrm{deg}$. The width of the annulus was always $3.52 \mathrm{deg}$. The one control and five disparity conditions were the same as those used in Experiment 3.

\section{Procedure}

Each of the three lateral separations was presented for four trials under each of the six viewing conditions, for a total of 72 trials. In all viewing conditions, the apparent size of the annulus was constant. Clarity ratings were obtained following the procedure of Experiment 3.

\section{Results}

Figure 4 shows perceived clarity for the control and disparity conditions under the three values of lateral separation from the present experiment and the one value from Experiment 3 . With respect to the three values of separation from this experiment, a one-way analysis of variance for repeated measures indicated that there is a reliable effect of disparity condition $[F(5,15)=7.99, p<.001]$. Duncan's multiple range test revealed the following differences to be significant: the control condition and disparity condition $6 \mathrm{~min} 40 \mathrm{sec}$ vs. disparity conditions $20 \mathrm{~min}$ $0 \mathrm{sec}, 26 \mathrm{~min} 40 \mathrm{sec}$, and $33 \mathrm{~min} 20 \mathrm{sec}(\mathrm{p}<.01)$; the control conditions vs. disparity condition $13 \mathrm{~min} 20 \mathrm{sec}$ $(\mathrm{p}<.05)$. The results shown in Figure 4 reveal that perceived clarity was less when the annulus was presented with the Landolt $C$ in the equal depth condition than when the $\mathrm{C}$ occurred alone in the control condition. An asymmetry of interference following depth changes of the annulus also can be observed: Clarity ratings were greater when the annulus was positioned behind the Landolt $\mathrm{C}$, and less when the annulus was located in front of the $C$, relative to clarity ratings in the equal depth condition. Because Experiment 3 employed the same observers under essentially the same conditions as the present experiment, clarity ratings for the value of lateral separation employed in Experiment 3-namely, .90 degwere included in the present data analysis to assess the effect of changing lateral separation between Landolt $C$. and annulus from .90 through $2.43 \mathrm{deg}$. A one-way analysis of variance for repeated measures found that there is a significant effect of lateral separation $[F(4,12)$ $=10.29, \mathrm{p}<.001]$. Duncan's multiple range test indicated that the following differences are significant: the control group vs. lateral separations of $.90,1.39$, 1.91 , and $2.43 \mathrm{deg}(\mathrm{p}<.01)$; lateral separation of $.90 \mathrm{deg}$ vs. separations of 1.91 and $2.43 \mathrm{deg}(\mathrm{p}<.05)$.

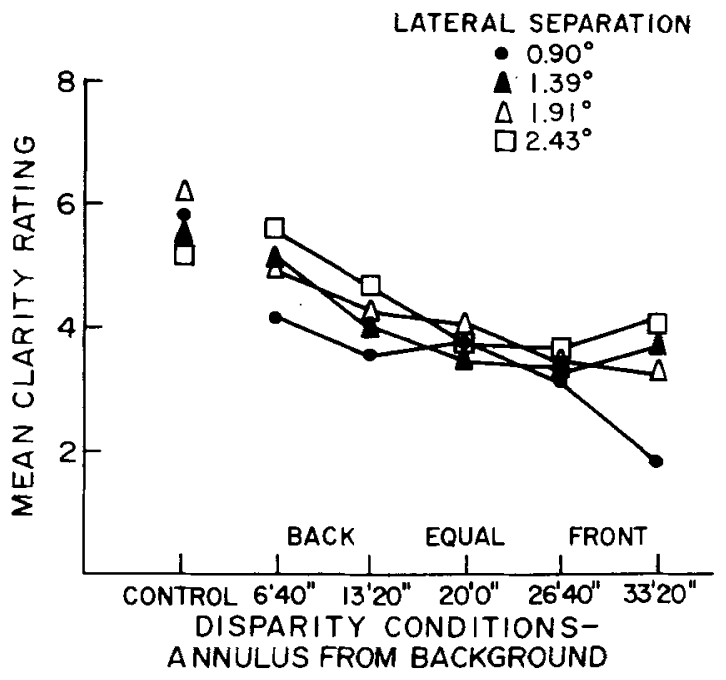

Figure 4. Clarity rating scores for the control condition, in which the Landolt $C$ test figure was presented without the annulus, and for five disparity conditions in which the Landolt $C$ and annulus were presented together (both Landolt $C$ and annulus were suprathreshold). "Back," “front," and "equal"' indicate the following: For disparity conditions 6 min $40 \mathrm{sec}$ and $13 \mathrm{~min} 20 \mathrm{sec}$, the annulus was positioned behind the Landolt $C$, and for disparity conditions $26 \mathrm{~min} 40 \mathrm{sec}$ and $33 \mathrm{~min} 20 \mathrm{sec}$, the annulus was positioned in front of the Landolt $C$. For disparity condition 20 min 0 sec, both Landolt $C$ and annulus occupied the same depth plane. Four values of lateral separation are shown, ranging in size from .90 (the smallest) to $2.43 \mathrm{deg}$ (the largest). Standard errors for Experiment 3, in which the value of separation was $.90 \mathrm{deg}$, fell within the range of .14 to .73 . For Experiment 4 , in which the values of separation were $1.39,1.91$, and $2.43 \mathrm{deg}$, standard errors fell within the range of .23 to .65 . 
Considering all values of separation, clarity ratings increased monotonically with the size of lateral separation between the inner edge of the annulus and the Landolt C; the smallest value of separation, .90 deg, received the lowest ratings, while the largest value, $2.43 \mathrm{deg}$, received the highest ratings.

\section{GENERAL DISCUSSION}

With respect to Experiment 1, the reduction in recognition accuracy when the Landolt $\mathrm{C}$ and annulus were in the same depth plane, compared with the control condition in which the $\mathrm{C}$ was presented without the annulus, demonstrates that the annulus did exert an inhibitory or interfering effect upon it. That interference, however, was clearly dependent upon the relative depth positions of the Landolt $\mathrm{C}$ and annulus: When the annulus was displaced in depth away from the observer and behind the Landolt $C$, recognition performance improved relative to that observed under the equal depth condition. Moreover, when the annulus was displaced in depth toward the observer and in front of the $\mathrm{C}$, performance worsened relative to that under the equal depth condition.

This pattern of results is similar to that observed by Lehmkuhle and Fox (1980) in their investigation of metacontrast masking, in which both annulus and test were transient stimuli. The two main conclusions derived from their study also apply equally well to the present results. First, interference is strongly influenced by depth position. Second, the influence is asymmetrical, following the pattern that Lehmkuhle and Fox called the front effect. The results of Experiment 1 suggest strongly that the occurrence of depth dependency and asymmetry is not restricted to the conditions of transient stimulation associated with visual masking.

In Experiment 1, the continual presence of the annulus and its manipulation in depth made it possible for eye movements to have played some role. However, the similarity between these results and those obtained in the Lehmkuhle and Fox experiments in which stimuli were presented too briefly to permit eye movements suggests that eye movements had no influence in Experiment 1.

In Experiment 3 both stimuli, Landolt $C$ and annulus, were continuously visible, and these are the conditions that most closely resemble any of the specific instances of lateral interference. Indeed, the same stimulus configurations, Landolt $\mathrm{C}$ and annulus, have been used in investigations of the crowding phenomena (e.g., Flom, Heath, \& Takahashi, 1963). Moreover, the dependent variable of Experiment 3, the rating of clarity, may correspond closely to an index of acuity as defined by the ability to resolve clearly an alphabet letter. The effects of the annulus and its position in depth on the clarity of the Landolt
$\mathrm{C}$ in Experiment 3 are similar in direction to those observed in Experiment 1 involving threshold recognition. Therefore, the results from Experiments 1 and 3 provide further support for the hypothesis that the dependency of interference on depth position, and the asymmetry of that dependency (i.e., the front effect), are not unique to threshold-level stimuli. Rather, the phenomena of depth dependency and asymmetry are observed generally in those situations for which the concept of lateral interference has been invoked.

The results of Experiments 2 and 4 were consistent with the hypothesis that perceptual interference, as measured by both threshold recognition and rated clarity, would decline as lateral separation between the Landolt $C$ and annulus increased. Taken together, the results of Experiments 2 and 4 suggest that lateral interference and the depth-dependent relationships observed in Experiments 1 and 3 come about when stimuli have small lateral separations or, in equivalent terms, when they have visual directions in close spatial proximity.

The more general conclusion supported by the results of all experiments is that the dependency of lateral interference on depth position, and the asymmetrical nature of that dependency (e.g., the front effect), are phenomena that can be generalized to more than one situation in which lateral interference is thought to occur. Evidence for depth dependency underscores the incompleteness of theories of interference based upon the concept of lateral inhibition and gives general support to the view that information about the location of objects in depth is processed either prior to, or simultaneously with, information about the characteristics of individual stimuli.

The asymmetrical nature of the depth dependencythat is, the front effect-is a new observation that does not readily fit into existing theoretical frameworks. Lehmkuhle and Fox (1980) suggested that when stimuli are close together in space or in time, the one closest to the observer receives in some sense preferential processing, or is given greater weight by the perceptual system. They conjecture that it would have been adaptive for the visual system to have evolved such a positive bias for the closest stimulus. On that view, the stimulus closest to the observer, in almost every situation, is the one demanding the greatest attention and the most immediate response.

A slightly different way of conceptualizing the front effect is to note its strong family resemblance to the figure-ground phenomena studied by the Gestalt psychologists. Whenever a stimulus configuration is perceived as a figure, it is always seen to be in front of, or on top of, the ground, and hence closer in depth to the observer than the corresponding ground. In addition to this perceptual dominance, the figure is also endowed with other perceptual advantages. 
For instance, as Koffka (1935) points out, the threshold for detecting a dim light flash is lower when it is imposed upon the figure rather than on the ground, even though the phýsical conditions of stimulation are identical in both cases.

The results found in the present series of experiments, together with the work of Gogel (e.g., 1978), suggest that many theories designed to account for perceptual interactions in two dimensions must undergo extensive elaboration and modification to provide an adequate account for perception in three dimensions.

\section{REFERENCES}

Bridgman, C. S. Analysis of a recently discovered stereoscopic effect. American Journal of Psychology, 1964, 77, 138-143.

Connsweet, T. N. Visual perception. New York: Academic Press, 1970.

Flom, M. C., Heath, G. G., \& Takahashi, E. Contour interaction and visual resolution: Contralateral effects. Science, 1963, 142, 979-980.

Fox, R., Lenmkuhle, S. W., \& Bush, R. C. Stereopsis in the falcon. Science, 1977, 197, 79-81.

Fox, R., Lehmkuhle, S., \& Leguire, L. E. Stereoscopic contours induce optokinetic nystagmus. Vision Research, 1978, 18, 1189-1192.
Gilchrist, A. When does perceived lightness depend on perceived spatial arrangement? Perception \& Psychophysics, 1980, 28, 527-538.

Gogel, W. C. The adjacency principle in visual perception. Scientific American, 1978, 238, 126-139.

Gulick, W. L., \& Lawson, R. B. Human stereopsis. New York: Oxford University Press, 1976.

Julesz, B. Foundations of cyclopean perception. Chicago: University of Chicago Press, 1971.

JuLEsz, B. Global stereopsis: Cooperative phenomena in stereoscopic depth perception. In R. Held, H. W. Leibowitz, \& H.-L. Teuber (Eds.), Handbook of sensory physiology (Vol. 8): Perception. New York: Springer-Verlag, 1978.

KoFFKA, K. Principles of Gestalt psychology. New York: HarcourtBrace, 1935.

LEH M KUHLE, S. W., \& Fox, R. The effect of depth separation on metacontrast masking. Journal of Experimental Psychology: Human Perception and Performance, 1980, 6, 605-621.

Shetty, S. S., Brodensen, A. J., \& Fox, R. System for generating dynamic random-element stereograms. Behavior Research Methods \& Instrumentation, 1979, 11, 485-490.

Staller, J. D., Lappin, J. S., \& Fox, R. Stimulus uncertainty does not impair stereopsis. Perception \& Psychophysics, 1980, 27, 361-367.

WooDworth, R. S. Experimental psychology. New York: Holt, 1938.

(Manuscript received October 27, 1980;

revision accepted for publication September 28, 1981.) 